\title{
Raw Video Summarization Based on Camera Motion and Alert System

\author{
Vaibhavi Patil ${ }^{1}$, Madhura Gawande ${ }^{1}$, Mayuri Patil ${ }^{1}$, \\ Tazeen Khan ${ }^{1}$, Mrs. Nilufar Zaman ${ }^{2}$ \\ ${ }^{1}$ Student, ${ }^{2}$ Professor \\ Department of Computer Engineering
} \\ Modern Education Society's College of Engineering, Pune, Maharashtra, India
}

\begin{abstract}
CCTV ordinance and its mechanism are progressively being used in joint facilities and organizations, as part of an attempt to thwart commonplace criminal acts. The environments monitoring has been expanded to protect residents in places, such as elementary schools and other care facilities, and city parks and ATM system.
\end{abstract}

The installation of the CCTV helps prevent crime and may aid in the solution of cases. Its role is also increasing in various forms. In addition, CCTV has been used for purposes, such as crime avoidance and the detection, influenced by the need for increased security.

In this paper we collectively recapitulate and interpret the different methodologies, used for detection, tracking and analyzing the objects within the images for any unusual activity that also used for public safety and other high security needed areas.

KEY WORDS: CCTV Installation, Open CV, Motion detection, block based background subtraction, Video Summarization

\section{INTRODUCTION}

Nowadays security is the most important issue arising due to increase in criminal acts such as child-related sexual offenses or commonplace criminal acts, to protect residents in places, and places that require high security like bank lockers, ATM centers, museum, and other care facilities. Video surveillance is a significant application that facilitates in examine region which needs high security. The scheme is used in detecting, analyzing, and tracking any unusual activity also it is used for public safety and other high security needed areas.

The installation of the CCTV helps prevent crime and may aid in the solution of cases. Its role is also increasing in various forms. The most important technique of this smart CCTV related research is to track and analyze objects within the images.

Motion Detection and Video Surveillance System Using IP camera is a system which helps in analyzing and tracking the objects and taking the required action accordingly. This System helps in providing security which reduces the human need and reduces labor. The System is best suited for indoor security as we are monitoring a particularly high-security area.

Motion Detection and Video Surveillance System Using IP camera is a System that helps in keeping the record of the activities and tracking the records whenever required.

There are various Smart CCTV technologies, using attached sensors that judges the situation and notifies the administrator directly or immediately responds. Additionally, it takes a simple picture of an image; this basic feature of CCTV has been studied extensively. The most important technique of this smart CCTV related research is to track and analyze objects within the images. The core technology of smart CCTV analysis lies in detecting, analyzing, and tracking the object's motion. However, the object, which is the target to be traced, can vary, depending on the situation, such as image size, orientation, and location, within consecutive frames. In addition, when 
the light's color or direction changes, it is difficult to trace the object, as it is perceived as another object, even though it is the same object as in the previous frames. Video analysis and video surveillance are active areas of research. The key areas are videobased detection and tracking, video-baseperson identification, and large-scale surveillance systems.

\section{LITERATURE REVIEW}

This paper [1] presents a framework for classification and identification of human behavior in complex motion. A template matching based technique for categorizes the objects and a rule-based approach to identify human activities are used respectively. Initially, moving objects are distinguished and their outline are generated in every frame, then categorize the generated outline with template matching based approach and finally rule-based classifier can categorize the human activities like running, walking, bending, boxing and jogging etc. This particular scheme can identify seven types of primal actions with high precision.

The paper gives an Automatic Video Summarizing Tool (AVST) for a personal video recording. The MPEG-7 visual descriptors are used to create a video index for a summary. The final index gives a preview of a movie along with non-linear access with thumbnails. With the help of this index searching of shots similar to a desired one within saved video sequences is possible. [2].

Visual analysis of video data can be time-consuming and prone to errors after long hours of observations so in this paper, author used video summarization algorithms, for analysis of video which can be classified into three categories: fast forwarding, key frame selection and frame re-composition [3][4].

Close Circuit Television Camera (CCTV) has played the significant role in many supervision and security scheme. The paper gives motion detection technique, object tracking methods and activity analyses for detection of suspicious actions. The security personnel will be provided with the accurate location of such event in order to analyze and act as soon as possible. With the help of Background, subtraction system can automatically record the whole enemy action. [5]

A new way for similarity search for human trajectories by means of a distance transform of every extracted human tunnel is explained in [6]. A proficient technique is used for scheming a direct distance transform (DDT). The technique first generates a distance transform from obtained trajectory information after that a similarity between the trajectory model and human trajectories are ranked. The system gives the human tunnels with trajectory information for analysis.

The author proposes a video visual analytics system for analyzing different views of information associated with moving objects in a video. Here sViSIT, tool is used to make smooth progress of browsing throughout the video content by interactive querying and retrieval of data. [7]

The idea of multiscale spatiotemporal tracking is explained by Arun Hampapur et al.[8] by using realtime video analysis, active cameras, multiple object models, for providing complete situational awareness.

Yongil Cho et al.[9] gives a collaborative inference framework for visual sensor networks and an efficient occupancy reasoning algorithm that is essential in elegant video surveillance systems. This framework is important where autonomous scene analysis is executed using distributed and collaborative processing among camera nodes with no need for a high-performance server. The framework Real Time Tunnel Based Video Summarization using Direct Shift Collision Detection (DSCD) is explained by Siriwat Kasamwattanarote et al. in [10] for summarizing a surveillance video. By organizing each human activity into the separated tunnel. An individual tunnel gives the information such as time, moving speed, direction, and moving trajectory identifying a group of similar activities.

Chen et al. [11] proposed a novel distance function labeled as edit distance on real sequences (EDR). EDR removes noise then alter one trajectory to another before measure. Thus, this technique enhances the correctness particularly with the trajectories having Gaussian noise.

\section{PROPOSED SYSTEM}

This project use Open CV library to capture camera images and identify interference using comparison block based motion object detection method. After comparison is done and an intrusion is found, it sends the streamed video from server to administrator 
computer. Admin can then take suitable action and alert local security.

Figure 1 shows the architecture illustration of the given scheme. The administrator can control the remote cameras using the following features:

$>$ Image Comparison and Intrusion detection comparison- block-based motion object detection method.

$>$ The mobile numbers for all the manager s/ owners who need to be contacted in case of an urgent situation are stored.

$>$ The scheme plays an alarm after identifying suspicious activity.

$>$ The scheme plays an alarm after identifying suspicious activity.

$>$ The scheme keeps track/monitor of all the activities. Hence a detailed record of messages received is sustained along with all the activities is also maintained.

The different modules involved in the system are as follows:

1. Capture Image: This module will take the camera ID as input and give the image as output.

2. Capture Video: This module will also take a camera ID as input and give the captured video as output.

3. Compare Image: In this module captured image is being taken as input and are compared which gives a difference between the captured image and the template image.

4. Video Summarization: In this module, the video is taken as input and detect motion from the video then crop this video and add into the new summarized video.

\section{ALGORITHM USED}

\section{Motion detection using block based background subtraction image.}

\section{A. Motional Region Detection Structure:}

Here we present a new technique such as BSM for motion detection. That is, it uses the subtraction of the recent frame image and the background image. The background image used at this time is not a background image prepared in advance. However, it creates the background screen in real-time when video shooting. The study of this technique followed into three steps:

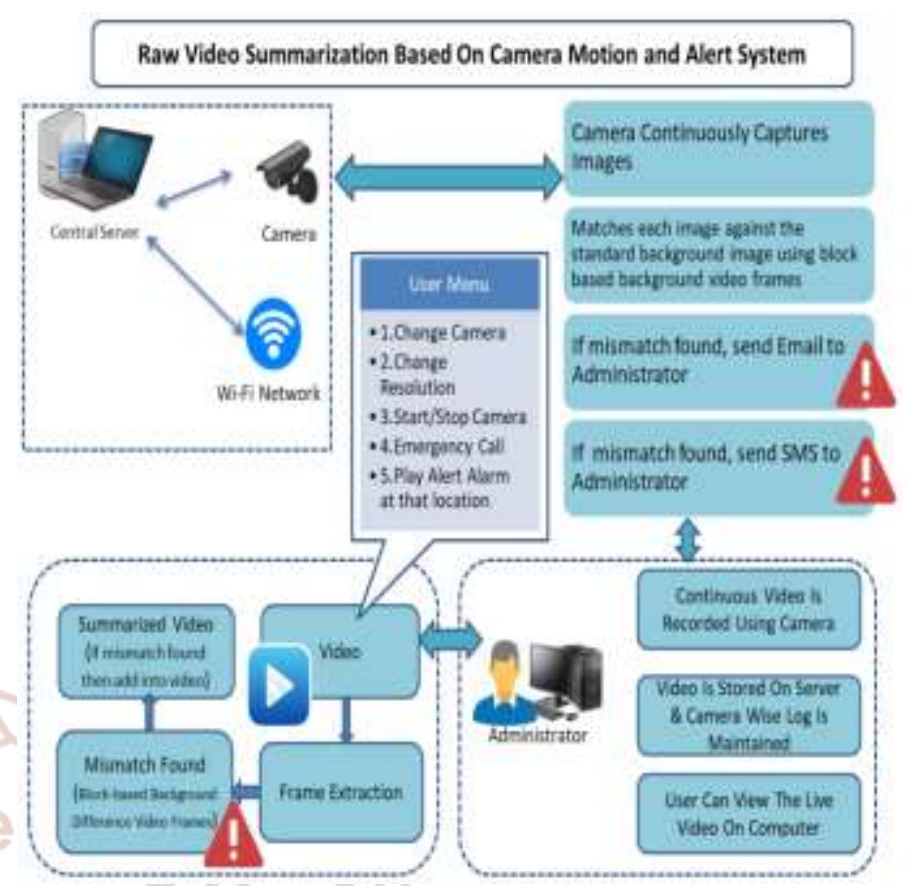

Fig1. Architecture Diagram

a. Blocking the input image and pre-processing the image by block zoning

b. Getting the difference image between the background image and block zoning.

c. Updating the background image

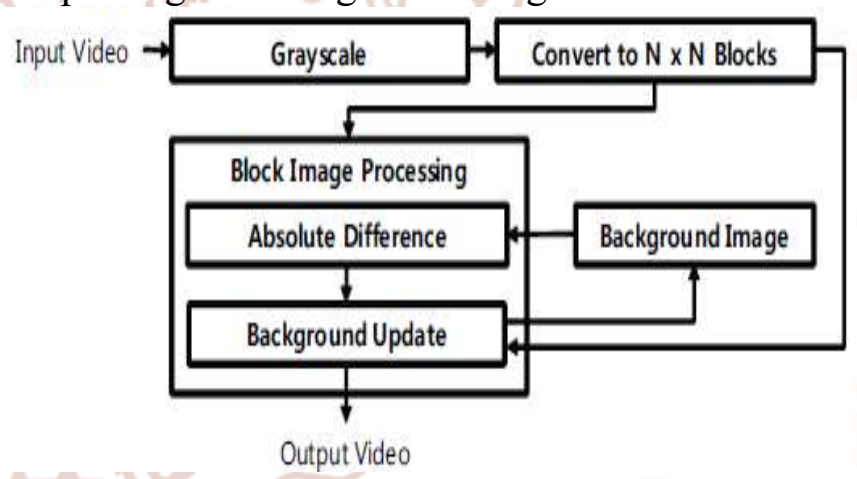

As shown in the above figure, the primary input image is a TV input means given in the form of NTSC model. It is the YIQ technique. It can be transformed into to grayscale with the help of following formula. Herein, F corresponding to the frame image, and $r, g$, b point towards Red, Green, Blue value, correspondingly, to the pixel subsequent to the position of $\mathrm{x}$ and $\mathrm{y}$.

$$
\begin{aligned}
G(x, y) & =0.299 \times F_{r}(x, y)+0.587 \times F_{g}(x, y) \\
& +0.114 \times F_{b}(x, y)
\end{aligned}
$$

The images we are getting are converting to grayscale and split into the square block with the whole number of pixels, N. consequently, the complete different image of the block is divided in the front using formula. 


$$
\begin{aligned}
& D_{n}(x, y)= \begin{cases}1, & \left|W_{n}(x, y)-B_{n}(x, y)\right|>t_{T} \\
0, & \text { otherwise }\end{cases} \\
& (x, y=0,1,2, \ldots \ldots, N-1 \quad N ; \text { window block size })
\end{aligned}
$$

In above formula, $\mathrm{n}$ represents the number of blocks, $\mathrm{W}$ the block corresponding to the existing image, $\mathrm{B}$ the block keep in touch with the background image, and $\mathrm{D}$ the value of the complete difference between $\mathrm{W}$ and $\mathrm{B}$ complete difference between $\mathrm{W}$ and $\mathrm{B}$.

\section{B. Background Image Update:}

Step 1: One-dimensional array is stated to accumulate every difference image luminance variance rate by block R (n), and start with to 0 . This step is performed only once throughout the first run.

Step 2: Integer variable $C$ to calculate the level of the transform for the total block is confirmed and initialized into 0 . Here in, $\mathrm{C}$ indicate the number of blocks. For the block difference image (Dn). Steps 3 and 4 are performed repeatedly.

Step 3: The number of pixels that have 1 as a value within the block difference image /(Dn) is put together. At this time, the sum of pixels represents the change in the luminance within the block. If it is equal to or greater than $t$, it is considered to have a change in the movement in the block, and the value of $\mathrm{R}(\mathrm{n})$ increases by 1 . In addition, the value of $\mathrm{C}$ increases by 1. Conversely, if the total of the pixels is smaller than t. we consider there is no change, the value of $R(n)$ reduces by 1 , and all the values of $\mathrm{Dn}$ are initialized to 0 . The image having no change in the intensity value in the block is initialized into 0 to remove noise. Herein, $\mathrm{t}$ utilizes a random threshold value i.e. block size $\mathrm{N}$.

$$
R(n)= \begin{cases}R(n)+1, & C=C+1, \quad \sum_{k=0}^{N^{2}} D_{n}(k)>\Delta t \\ R(n)-1, & D_{n}=0, \ldots, 0, \text { otherwise }\end{cases}
$$

Step 4: In above formula, if the value of $R(n)$ is less than ' -1 ', the background image of the block is updated. Otherwise, it is not updated and remains as the previous background image.

\section{CONCLUSION}

CCTV is used in various places like in government areas, in government offices for security purpose. The presented system can judge the current situation by detecting motion in real-time by analyzing the behavioral patterns of the objects and its association with the surrounding environment.
Detection of motion is essential to identify the activities performed by an object and record the activities performed by video recording. In addition, the system is able to give an abstract view of the entire video to reduce the time consumed to watch the whole video.

\section{REFERENCES}

1. A K Singh Kushwaha, O Prakash, A Khare, Maheshkumar H Kolekar, "Rule based human activity recognition for surveillance system", 4th IEEE Int. Conf. on Intelligent Human Computer Interaction, India, 2012.

2. Lee, Jae-Ho, Gwang-Gook Lee, Whoi-Yul Kim, "Automatic video summarizing tool using MPEG7 descriptors for personal video recorder", IEEE Transactions on Consumer Electronics 49.3, pp. 742-749, 2003.

3. Po Kong Lai, Marc Decombas, Kelvin Moutet, Robert Langaniere ,"Video summarization of surveillance camera" : 2016 13th IEEE International Conference on Advanced Video and Signal Based Surveillance (AVSS).

4. Shayok Chakraborty, Omesh Tickoo and Ravi Iyer "Adaptive Keyframe Selection for Video Summarization" 2015 IEEE Winter Conference on Applications of Computer Vision.

5. Massimo Piccardi, "Background subtraction techniques: a review", IEEE International Conference on Systems, Man and Cybernetics , 10-13 Oct. 2004.

6. Nagul Cooharojananone1,

Siriwat Kasamwattanarote1, Shin'ichi Satoh2, and Rajalida Lipikorn "Real Time Trajectory Search in Video Summarization using Direct Distance Transform",(C2010 IEEE.

7. Amir H. Meghdadi, Pourang Irani, "Interactive Exploration of Surveillance Video through Action Shot Summarization and Trajectory Visualization", IEEE TRANSACTIONS ON VISUALIZATION AND COMPUTER GRAPHICS, VOL. 19, NO. 12, DECEMBER 2013.

8. Arun Hampapur, Lisa Brown, Jonathan Connell, Ahmet Ekin, Norman Haas, Max Lu, Hans Merkl, Sharath Pankanti, Andrew Senior, Chiao-Fe Shu, and Ying Li Tian, "Smart Video Surveillance, Exploring the concept of multiscale 
spatiotemporal tracking", IEEE SIGNAL Kasamwattanarote, Nagul Cooharojananone, PROCESSING MAGAZINE. MARCH 2005. Satoh Shin'ichi, and Rajalida Lipikorn, "Real

9. Yongil Cho; Sang Ok Lim; Hyun Seung Yang "Collaborative occupancy reasoning in visual sensor network for scalable smart video surveillance", IEEE Transactions on Consumer Electronics (Volume: 56, Issue: 3, Aug. 2010.

10. Yongil Cho ; Sang Ok Lim ; Hyun Seung Yang "Collaborative occupancy reasoning in visual Time Tunnel Based Video Summarization using Direct Shift Collision Detection," in Proceedings of PCM, Shanghai, China, 2010, in press.

11. Lei Chen, Tamer M. Özsu, and Oria Vincent, "Robust and Fast Similarity Search for Moving Object Trajectories," in Proceedings of ICMD, 2005, pp. 491 - 502.

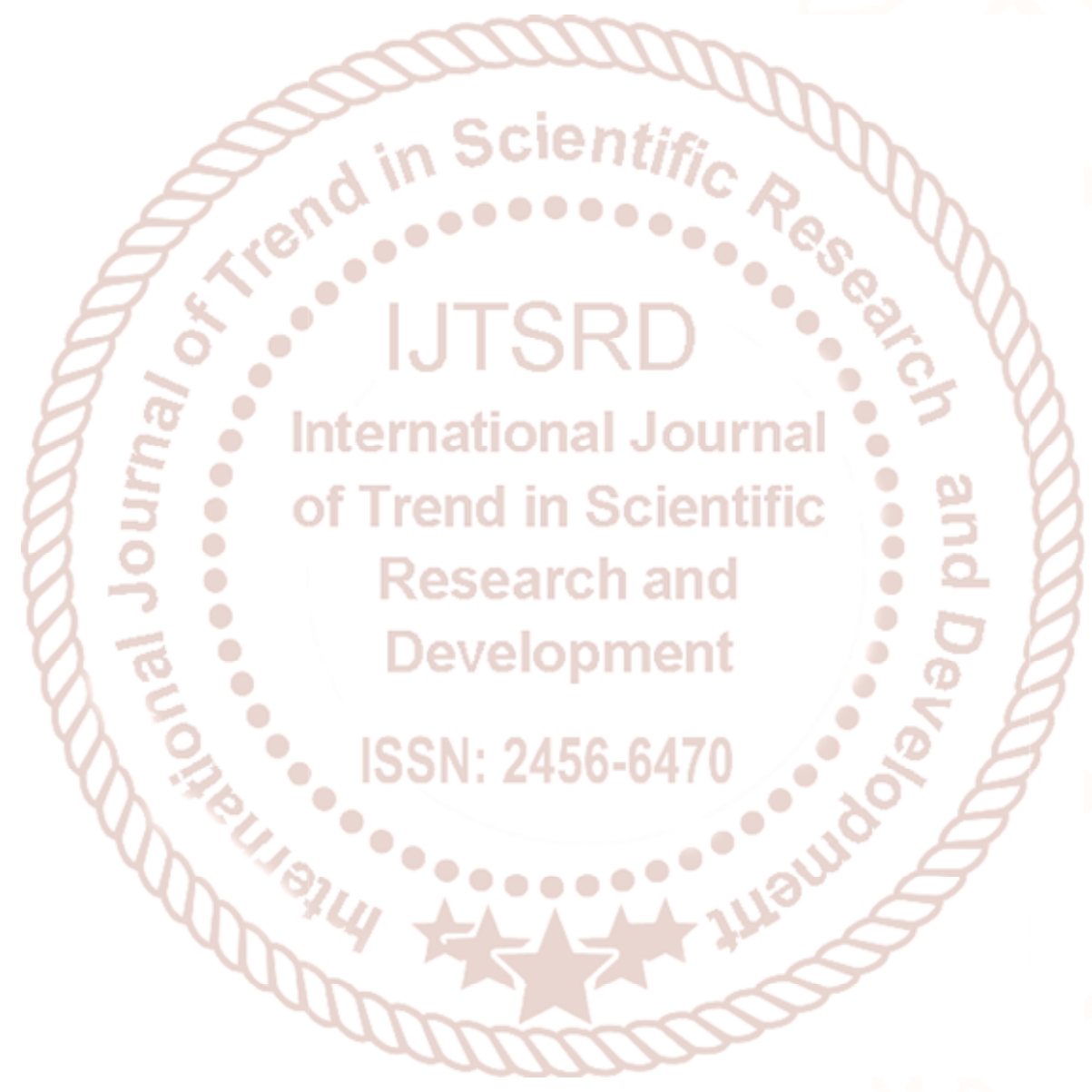

